\title{
Prevalência de Transtorno Mental e Comportamental em Policias Militares/SC, em Licença para Tratamento de Saúde
}

\author{
Prevalence of Mental and Behavioral Disorders \\ in Military Police of Santa Catarina
}

Prevalencia de los Trastornos Mentales y del Comportamiento en Policías Militares/SC con Licencia para Cuidado de la Salud

Fabíola Polo de Lima, Vera

Lúcia Guimarães Blank \&

Fabricio Augusto Menegon

Universidade Federal

de Santa Catarina

http://dx.doi.org/10.1590/1982-3703002242013 
Resumo: Este estudo estimou a prevalência de Transtorno Mental e Comportamental (TMC) em Policiais Militares em Licença para Tratamento de Saúde (LTS), da região metropolitana de Florianópolis/SC, casos notificados pela Junta Médica. Caracteriza-se como transversal descritivo, cuja variável dependente é o TMC, categorizado em sim e não. As variáveis exploratórias referem-se às características demográficas (sexo, faixa etária) e ocupacionais (tempo de serviço, batalhão de atuação, graduação/posto e hierarquia). As análises univariadas incluíram frequências relativas e absolutas, utilizando o teste do Qui-Quadrado, teste do qui-quadrado para tendência e o Fisher Exact. Para verificar a associação de TMC com as variáveis exploratórias, foram construídos modelos de regressão logística multivariada e hierárquica. Foram testadas as associações entre as variáveis dependentes e cada uma das variáveis independentes por meio do Teste do Qui-Quadrado de Pearson $(\chi 2)$. Sexo e hierarquia entraram na modelagem. A análise verificou prevalência de TMC de $24 \%$ e fator de associação positiva com TMC e hierarquia. Os resultados demonstram alta prevalência de licença para tratamento de saúde por TMC e dados que sugerem estudos mais específicos para fundamentar estratégias de prevenção e enfrentamento ao TMC, buscando preservar e melhorar a saúde psicológica desta categoria profissional.

Palavras-chaves: Prevalência. Polícia Militar. Saúde Mental. Saúde do Trabalhador.

Abstract: This study estimated the prevalence of Mental and Behavioral Disorders (MBD) in military police for the License for Health Care (LHC) in the metropolitan region of Florianópolis/SC, cases registered by the Police Medical Board. For this study, the dependent variable was MBD and categorized as yes or no. Independent variables refer to demographic characteristics (gender, age) and occupational (service time, as battalion, graduation/post and hierarchy). The univariate analysis included absolute and relative frequencies, the chi-square test, the chi-square test for trends, and Fisher's Exact. To investigate the association of MBD with the individual characteristics, multivariate and hierarchical logistic models were created. Associations between the dependent variables and each of the independent variables through the Pearson chi-square test $(\chi 2)$ were investigated. The model included sex and hierarchy. The analysis found the prevalence of Mental and Behavioral Disorders to be $24 \%$ and positive association factor between MBD and hierarchy. The results indicate a high prevalence of sick leave treatment for MBD and additional data which suggest that more specific studies are needed to support prevention strategies and coping of MBD, to preserve and improve the psychological health of this profession.

Keywords: Prevalence. Military Police. Mental Health. Occupational Health.

Resumen: Este estudio estima la prevalencia de trastornos mentales y del comportamiento (TMC) en la Policía Militar con Licencia para el Cuidado de la Salud (LTS), de la región metropolitana de Florianópolis/SC, casos reportados por la Junta Médica. Se caracteriza por ser transversal descriptivo, cuya variable dependiente es el TMC, categorizado como sí y no. Las variables exploratorias se refieren a las características demográficas (sexo, edad) y (tiempo de servicio, en calidad de batallón, graduación/post y jerarquía) ocupacional. El análisis univariado incluyó frecuencias absolutas y relativas, mediante la prueba de chi cuadrado, prueba de chi-cuadrado para la tendencia y Fisher exacta. Para comprobar la asociación TMC con las variables explicativas se construyeron modelos de regresión logística multivariada y jerárquica. Se evaluaron las asociaciones entre las variables dependientes y cada una de las variables independientes a través de la prueba de chi-cuadrado de Pearson ( $\chi 2$ ). El sexo y la jerarquía entraron en el modeloo. El análisis encontró prevalencia de TMC de $24 \%$ y factor de asociación positiva con TMC y jerarquía. Los resultados muestran una alta prevalencia de licencia para tratamiento de la salud por TMC y datos que sugieren estudios más específicos para apoyar las estrategias de prevención y enfrentamiento de TMC, tratando de preservar y mejorar la salud psicológica de esta categoría profesional.

Palabras clave: Prevalencia. La Policía Militar. La Salud Mental. La Salud Ocupacional. 


\section{Introdução}

Segundo estimativas da Organização Mundial de Saúde (OMS), 83 milhões de pessoas no mundo, com idade entre 18 e 65 anos, são afetadas por algum tipo de transtorno mental. Estudos realizados na União Europeia, Islândia, Noruega e Suíça, demonstram que $27 \%$ da população adulta apresentou algum tipo de transtorno mental no ano de 2010, sendo as taxas mais elevadas nas mulheres para quase a totalidade dos transtornos. Esses números vêm ao encontro de estimativas globais, apresentando taxa de $33,2 \%$ para as mulheres e taxa de $21,7 \%$ para os homens (WHO, 2011).

Calcula-se que cerca de 340 milhões de pessoas no mundo inteiro sejam afetadas por depressão, 45 milhões por esquizofrenia e 29 milhões por demência. Os transtornos mentais respondem por uma proporção elevada de todos os anos de vida perdidos em função de uma deficiência ou transtorno, e a previsão é de que esse ônus cresça significativamente no futuro (WHO, 2001). Os Transtornos Mentais e Comportamentais (TMC) são alterações clínicas e comportamentais com funcionamento prejudicado ou associação destes e podem causar expressivo sofrimento e danos em áreas do funcionamento mental, resultante de fatores orgânicos, sociais, genéticos, químicos ou psicológicos (VandenBos, 2010).

1 A CID-10 foi articulada para ser uma classificação nuclear relacionadas à doença e à saúde.
A Classificação Internacional de Doenças - $10^{a}$ revisão - CID $10^{1}$ (Organização Mundial da Saúde, 2000) define TMC no seu capítulo $\mathrm{V}$, com os códigos definidos pela letra $F(C I D F)$ compreendidos entre F00 ao F99, com uma ampla categorização, baseado em condições clínicas e alguns preceitos de diagnóstico. Nesta classificação são apresentadas as seguintes subdivisões para TMC: transtornos mentais orgânicos, inclusive os sintomáticos; transtornos mentais e comportamentais devidos ao uso de substância psicoativa; esquizofrenia, transtornos esquizotípicos e transtornos delirantes; transtornos do humor (afetivos); transtornos neuróticos, transtornos relacionados com o stress e transtornos somatoformes; síndromes comportamentais associadas a disfunções fisiológicas e a fatores físicos; transtornos da personalidade e do comportamento do adulto; retardo mental; transtornos do desenvolvimento psicológico; transtornos do comportamento e transtornos emocionais que aparecem habitualmente durante a infância ou a adolescência; transtorno mental não especificado. Estudos internacionais apontam problemas de saúde mental relacionados ao trabalho, como a pesquisa realizada em uma empresa pública na Alemanha, onde oTMC surge como responsável pela segunda maior causa de afastamento (Browers, Terluin, Tiemens, \& Verhaak, 2009; Roelen, Koopmans, Anema, \& Beek 2010).

Nos diagnósticos de Afastamento para Licença para Tratamento de Saúde (LTS), os transtornos mentais são responsáveis por uma considerável parcela, diante disso, deve-se considerar a necessidade de verificar as condições de trabalho devido estar relacionado aos problemas que incapacitam os trabalhadores (Cruz, 2010). Os TMC ocasionam consequências sociais e econômicas para o próprio sujeito e para a sociedade como um todo. O afastamento do trabalhador para o tratamento da saúde requer atenção pelo papel que o trabalho exerce na vida da pessoa, pois é uma das estruturas da constituição do sujeito, influenciando a construção da identidade da pessoa. (Mendes, Ghizoni, \& Araújo, 2011).

O uso da epidemiologia para estudos sobre saúde mental e trabalho deve-se à ascensão do campo da Saúde Coletiva no Brasil, através de estudos epidemiológicos para exploração dessa temática. Para pensar nessa relação através da epidemiologia, deve-se estudar a sua complexidade e, como afirma o autor, "o trabalho e seus elementos constituintes são estruturantes do conteúdo e da forma do sofrimento apresentado" (Mendes, Ghizoni, \& Araújo, 2011, p. 331).

A produção de conhecimento a partir da interface da Saúde do Trabalhador com a Saúde Coletiva, especificamente a Epidemiologia, contribui tanto para a identificação de fatores e condições que influenciam a saúde das pessoas em um dado processo produtivo que podem gerar doenças ocupacionais relacionadas a 
diversas patologias (Asmus, Meyer, \& Castro, 2009), quanto para o desenvolvimento de ações para prevenir tais problemas de saúde com uma abordagem interdisciplinar.

Estudo sobre as tendências da produção científica em Saúde do Trabalhador no Brasil verificou um total de 1.025 produções até o ano de 2004. Há uma crescente produção científica sobre a temática no decorrer dos anos, sendo encontrados sete estudos datados antes de 1970 e 154 estudos entre os anos de 1970 e 1989. E um aumento significativo a partir do ano de 1990, com 333 estudos publicados. Este mesmo número se repetiu para os anos de 2000 e 2004. Apenas a partir de 1990 acontece o aumento de estudos sobre doenças crônicas, dentre essas, as doenças mentais (de quatro estudos encontrados entre os anos de 1980 a 1989, passaram para 23 em 1990) (Santana, 2006).

Estudo sobre o adoecimento de Servidores Públicos Estadual demonstra que no ano de 2005 o afastamento por TMC foi responsável por $17.8 \%$ das ocorrências, está em primeiro lugar dos diagnósticos que determinaram a concessão da Licença para Tratamento de Saúde (Campos, 2006). Em dados atuais, trabalhadores da iniciativa privada, apresentaram aumento de $28 \%$ nos benefícios concedidos devido ao diagnóstico deTMC (Brasil, 2014a) e, entre os meses de janeiro a abril de 2014, foi a quarta causa de afastamento para tratamento de saúde (Brasil, 2014b).

O estresse está diretamente relacionado aos transtornos mentais, seja direta ou indiretamente. Mapeamento realizado em profissionais da segurança pública do Estado de Santa Catarina (2010) revela que a necessidade em realizar horas-extras para complementar a renda aparece como principal estressor. Dentre os sinais e sintomas de estresse que os participantes relataram, como a tensão muscular, sensação de desgaste constante, insônia, etc., a percepção de cansaço excessivo é mais evidente em policiais militares. Um expressivo número de indivíduos que referiram sinais e sintomas encontram-se em exaustão e quase-exaustão, ou seja, indícios de um provável transtorno mental e comportamental.
Os Policiais Militares (PM) possuem características específicas, em razão do seu objeto e objetivo de trabalho, caracterizando-se por ser um trabalho da área de setor de serviços e com a missão de assegurar o cumprimento da lei por todos os cidadãos. O setor de serviços é uma área pouco pesquisada, mesmo sendo responsável por praticamente dois terços do emprego no Brasil e sua considerável responsabilidade pelos indicadores econômicos (Melo et al. apud Minayo, Souza, \& Constantino, 2007).

A origem da atividade da Polícia Militar é a segurança pública, e eles são, igualmente, servidores públicos amparados pela Constituição Federal, ou seja, têm o direito constitucional a resguardar o físico e mental no exercício do trabalho (Minayo, Souza \& Constantino, 2008). Considerando os aspectos legais, inerentes ao dia a dia do policial militar, como a frequente relação com a violência e a desordem, também se deve considerar os aspectos organizacionais da instituição, a qualidade das relações a que estão submetidos dentro da organização (Castro, 2012). Minayo et al. (2007) reforçam essa condição: "risco [...] representa uma condição intrínseca à profissão policial" (p. 2768).

Fatores de risco específicos dos policiais contribuem para o adoecimento como insalubridade, perigos ambientais, rotinas administrativas e operacionais. A dedicação integral exigida do policial, tempo este que perpassa sua vida pessoal e exige estar alerta vinte quatro horas por dia, mesmo em período de folga, ocasiona mudanças de hábitos, pois o seu compromisso é estar a serviço da lei e da ordem pública mesmo em horário de folga (Minayo, Souza, \& Constantino, 2007; Souza, \& Minayo, 2005). Além disso, o constante convício com a violência e criminalidade e o uso permanente da arma de fogo (Andrade, Souza, \& Minayo, 2009).

Grande parte dos estudos com policiais militares são relacionados à temática do estresse (Bezerra, Minayo, \& Constantino, 2013; Costa et al. 2010; Ferreira, Augusto, \& Silva, 2008; Souza, Minayo, Silva \& Pires, 2012; Souza, Schenker, Constantino, \& Correia, 2013; Oliveira, \& Bardagi, 2010). 
Estudo sobre a atividade profissional do Policial Militar e a relação com a saúde mental, demonstrou três patologias com maior prevalência em internações hospitalares, realcionadas ao TMC: $61,0 \%$ transtornos mentais e comportamentais devido ao uso de álcool (CID F10), 33,3\% episódios depressivos (CID F32) e transtorno depressivo recorrente (CID F33), e com $11,7 \%$ a esquizofrenia (Mascarenhas, 2009). Outro estudo aponta que, no período entre 1995 e 1998, 41\% dos Policiais Militares da Paraíba de aposentaram por invalidez que foi motivada por questões relacionadas à saúde mental (Silva, \& Vieira, 2008).

A produção científica acerca do policial vem aumentando, no entanto, ainda é necessário expandir a produção científica, considerando a quantidade existente destes profissionais os estudos ainda são inexpressivos. O tema vem conquistando espaço no meio acadêmico, possibilitando fundamentar intervenções focadas em minimizar o efeito negativo do trabalho na saúde mental. Valorizar essa relação é pensar na importância do papel do policial militar sobre a sociedade (Silva, 2009).

Observa-se uma lacuna nos estudos sobre transtorno mental e o trabalho do policial militar de Santa Catarina e, nesse sentido, este estudo irá incrementar a discussão, fornecendo subsídios para a ampliação do debate desta temática. O uso da epidemiologia para estimar a prevalência de Transtornos Mentais e Comportamentais em Policiais Militares de Santa Catarina (Polícia Militar de Santa Catarina, 2011) favorecerá um olhar específico para esses trabalhadores, cujo processo produtivo é diferenciado, pois atuam diariamente de forma preventiva e repressiva em questões relacionadas à manutenção da ordem pública.

O objetivo deste estudo foi verificar a prevalência de transtorno mental e comportamental em Policiais Militares da região metropolitana de Florianópolis/SC em licença para tratamento de saúde, casos notificados pela Junta Médica no ano de 2012. Compreender essa dinâmica possibilitará ações específicas para promoção de saúde e prevenção de agravos desta categoria profissional.

\section{Método}

Trata-se de um estudo epidemiológico, descritivo de corte transversal. O critério de inclusão no estudo foi: a) Policiais Militares da Região Metropolitana de Florianópolis/SC afastados do trabalho em Licença para Tratamento de Saúde (LTS) no ano de 2012. E os critérios de exclusão foram: a) reincidência de afastamento pelo mesmo diagnóstico; b) ilegibilidade do prontuário médico; c) registros ou prontuários com dados incompletos.

A coleta dos dados foi autorizada pela Diretoria de Saúde e Promoção Social e pela Divisão de Serviço Social do Hospital da Polícia Mili$\operatorname{tar}$ (HPM). Foram coletados dados secundários por meio dos registros das LTS disponibilizados pela Junta Médica da Polícia Militar de Santa Catarina. Complementarmente, foram verificados os prontuários de saúde, sempre que havia conflito de informação nos registros. Estes dados encontram-se no HPM, localizado na cidade de Florianópolis/SC, direcionado aos funcionários da Secretaria de Segurança Pública e defesa do Cidadão, seus dependentes e população em geral.

Os dados foram computados em um protocolo de análise dos registros e prontuários, criado para investigar os quadros diagnósticos de TMC e as variáveis estudadas. Os dados coletados correspondem aos registros e prontuários referentes ao ano de 2012, escolha realizada por conveniência. Para padronização da coleta de dados foi considerado TMC de todos os diagnósticos contemplados no capítulo V, que estão apresentados através dos códigos F-00 até F-99, da CID-10. A seguir, os dados foram organizados e analisados com fundamentação da Saúde Coletiva, especificamente na Epidemiologia e na Saúde do Trabalhador. Para a codificação das informações no banco de dados, foram atribuídos valores numéricos para realização da análise estatística.

A variável dependente deste estudo é o Transtorno Mental e Comportamental que foi categorizado em sim e não. As variáveis exploratórias referem-se às características sócio-demográficas, como: sexo, faixa etária 
$24^{\circ}$ batalhão: Florianópolis (Centro); $21^{\circ}$ batalhão:

Florianópolis (Norte); $22^{\circ}$ batalhão: Florianópolis (Continente); CPGd: Companhia de Policiamento de Guarda; BCSv: Batalhão de Comando e Serviço; $7^{\circ}$ batalhão: São José; $16^{\circ}$ batalhão: Palhoça; $24^{\circ}$ batalhão: Biguaçú; GESA: Guarnição Especial de Santo Amaro; CRE/COPOM: Centro Regional de Emergência; BAPM; Batalhão de Aviação; BPMA: Batalhão da Polícia Ambiental; BOPE: Batalhão de Operações Especiais; CPM Pol. Cães: Centro de Policiamento com cães; GEPMMOn: Guarnição Especial de Polícia Militar Montada (Cavalaria); GECHq: Grupamento de Polícia de Choque; CEPM: Centro de

Ensino da Polícia Militar; Diretorias: Diretoria de Apoio Logístico, de Saúde e Promoção Social, de

Tecnologia e Sistemas de Informação), graduação (oficial e praça).
(18 a 29 anos, 30 a 39 anos, 40 a 49 anos e 50 a 59 anos); ocupacionais: tempo de serviço (6 meses a 4 anos, 5 a 10 anos, 11 a 15 anos, 16 a 20 anos, 21 a 25 anos e 26 anos ou mais), batalhão de lotação ( $4^{\circ}$ batalhão, $21^{\circ}$ batalhão, $22^{\circ}$ batalhão, CPGd, BCSv, $7^{\circ}$ batalhão, $16^{\circ}$ batalhão, $24^{\circ}$ batalhão, GESA, CRE/COPOM, BAPM, BPMA, BOPE, COM Polícia de Cães, GEPMMOn, GECHq, CEPM e Diretorias) e graduação (oficial e praça) ${ }^{2}$.

Os dados foram tabulados no software Excel, versão 2007, e as análises realizadas no programa SPSS versão 16.0. As análises univariadas incluíram as frequências relativas e absolutas. O teste do Qui-Quadrado, para as variáveis nominais, teste do Qui-Quadrado para tendência para as variáveis ordinais e o Fisher Exact para as variáveis nominais que em uma das caselas foi observado o número de observações menor que cinco, foram empregadas como medida de associação nas análises bivariadas. O nível de significância adotado foi de $5 \%$. As variáveis demográficas e ocupacionais foram consideradas pela data do primeiro diagnóstico. E a variável dependente, TMC, computado apenas uma vez.

\section{Modelo de regressão logística para a variável TMC}

Para verificar a associação de TMC com as variáveis sociodemográficas e ocupacionais, foram construídos modelos de regressão logística multivariada e hierárquica. A variável dependente ou desfecho foi a presença de diagnóstico de Transtorno Mental e Comportamental - TMC. As variáveis de associação foram compostas pelas demais variáveis coletadas e agrupadas de acordo com a referência anterior. O plano de análise dos dados utilizados para o desenvolvimento dos modelos foi da seguinte forma:

- A variável dependente TMC foi dicotomizada em "0 = SIM" e "1 = NÃO", conforme objetivo do estudo, em verificar a prevalência de TMC em Policiais Militares de Santa Catarina, em processo de Licença para Tratamento de Saúde, no ano de 2012;
- As variáveis independentes foram transformadas em variáveis categóricas com duas ou três categorias, sendo que os pontos de corte para a definição das categorias foram estabelecidos pela mediana dos dados ou por tercis $\left(1^{\circ}, 2^{\circ}\right.$ e $3^{\circ}$ tercil), de acordo com a característica da distribuição de frequência de cada variável;

- Foram testadas as associações entre as variáveis dependentes e cada uma das variáveis independentes por meio do Teste do Qui-Quadrado de Pearson $(\chi 2)$. As variáveis testadas em que a associação apresentou nível de significância de $p \leq 0,20$ foram selecionadas para entrada na modelagem de regressão logística;

- A ordem de entrada de cada variável no modelo aconteceu de maneira ascendente em relação ao valor de p obtido no Teste do Qui-Quadrado de Pearson $(\chi 2)$, isto é, variáveis com menor valor de $\mathrm{p}$ eram incluídas primeiro no modelo. Se o valor de $\mathrm{p}$ fosse igual para diferentes variáveis, a ordem de entrada dessas variáveis se dava a partir do maior valor do Qui-Quadrado obtido no teste, e assim sucessivamente;

- Para a variável dependente TMC foram elaboradas modelos de regressão logística univariada e multivariada;

- Todas as variáveis que apresentassem nível de significância de $\mathrm{p}<0,05$ e que o intervalo de confiança (IC95\%) não incluísse a unidade, foram mantidas no modelo final para a variável dependente.

\section{Escolha de variáveis para inclusão no modelo}

Por meio da Regressão Logística, foram testados os fatores associados ao TMC. Assim, obtiveram-se as Razões de Chance (OR) nas análises bivariadas e multivariável com seus respectivos intervalos de confiança (IC95\%). A partir dos resultados da primeira análise foram incluídas na análise multivariável todas as variáveis que apresentaram $p<0,20$, sendo associadas aquelas com $p<0,05$. O conjunto de dados para idade, tempo de serviço e batalhão foi agrupado com o objetivo de 
tornar a distribuição mais homogênea e as variáveis sexo e hierarquia entraram na modelagem. No modelo, hierarquia apresentou significância, ou seja, é fator de associação positiva com TMC e a variável sexo não apresentou significância.

\section{Resultados}

Características demográficas e vínculo com a Polícia Militar

A Tabela 1 mostra que a maioria dos policiais da região metropolitana de Florianópolis/SC, em LTS em 2012 é do sexo masculino (90\%), está na faixa etária dos 40 aos 49 anos (46\%) e com tempo de serviço entre 16 a 20 anos. Quanto à região de atuação, o $4^{\circ}$ Batalhão (Florianópolis-centro) apresenta o maior índice de policiais militares em LTS (14,5\%). Observou-se que houve um predomínio de afastamentos de soldados (61\%), confirmando o dado seguinte, em que a hierarquia que mais se afastou foi a de Praças (97\%).

Prevalência de transtorno mental e comportamental em Policiais Militares

A análise verificou prevalência de $24 \%$ de TMC, notificados, em Policiais da região metropolitana de Florianópolis/SC afastados para LTS, no ano de 2012 (Figura).

\section{Associação entre as} variáveis demográficas e de vínculo com a corporação

\section{com o transtorno mental e comportamental em Policiais Militares}

Através da Regressão Logística, testaram-se os fatores associados ao TMC. Assim, obtiveram-se as Razões de Chance (OR) nas análises bivariadas e multivariável com seus respectivos intervalos de confiança (IC95\%). A partir dos resultados da primeira análise foram incluídas na análise multivariável todas as variáveis que apresentaram $p<0,20$, sendo associadas aquelas com $p<0,05$.
Tabela 1. Características demográficas e de vínculo com a corporação em Policiais Militares da região metropolitana de Florianópolis/SC, afastados em licença para tratamento de saúde no ano de 2012.

\begin{tabular}{|c|c|c|}
\hline Variável & $\mathbf{N}$ & $\%$ \\
\hline \multicolumn{3}{|l|}{ Sexo } \\
\hline Masculino & 180 & 90,0 \\
\hline Feminino & 20 & 10,0 \\
\hline \multicolumn{3}{|l|}{ Faixa etária } \\
\hline 18 a 29 anos & 27 & 13,5 \\
\hline 30 a 39 anos & 69 & 34,5 \\
\hline 40 a 49 anos & 92 & 46,0 \\
\hline 50 a 59 anos & 12 & 6,0 \\
\hline \multicolumn{3}{|l|}{ Tempo de serviço } \\
\hline 6 meses a 4 anos & 17 & 8,5 \\
\hline 5 a 10 anos & 41 & 20,5 \\
\hline 11 a 15 anos & 10 & 5,0 \\
\hline 16 a 20 anos & 56 & 28,0 \\
\hline 21 a 25 anos & 39 & 19,5 \\
\hline 26 anos ou mais & 37 & 18,5 \\
\hline \multicolumn{3}{|l|}{ Batalhão de lotação } \\
\hline $4^{\circ}$ batalhão & 29 & 14,5 \\
\hline $21^{\circ}$ batalhão & 12 & 6,0 \\
\hline $22^{\circ}$ batalhão & 19 & 9,5 \\
\hline CPGd & 9 & 4,5 \\
\hline BCSv & 19 & 9,5 \\
\hline $7^{\circ}$ batalhão & 24 & 12,0 \\
\hline $16^{\circ}$ batalhão & 17 & 8,5 \\
\hline $24^{\circ}$ batalhão & 6 & 3,0 \\
\hline GESA & 4 & 2,0 \\
\hline CRE/COPOM & 14 & 7,0 \\
\hline BAPM & 7 & 3,5 \\
\hline BPMA & 4 & 2,0 \\
\hline BOPE & 8 & 4,0 \\
\hline CPM pol. cães & 2 & 1,0 \\
\hline GEPMMOn & 6 & 3,0 \\
\hline $\mathrm{GECHq}$ & 3 & 1,5 \\
\hline CEPM & 5 & 2,5 \\
\hline Diretorias & 12 & 6,0 \\
\hline \multicolumn{3}{|l|}{ Posto/Graduação } \\
\hline Soldado & 122 & 61,0 \\
\hline Cabo & 42 & 21,0 \\
\hline Sargento & 30 & 15,0 \\
\hline Tenente & 1 & 0,5 \\
\hline Major & 3 & 1,5 \\
\hline Tenente coronel & 2 & 1,0 \\
\hline \multicolumn{3}{|l|}{ Hierarquia } \\
\hline Oficial & 6 & 3,0 \\
\hline Praça & 194 & 97,0 \\
\hline
\end{tabular}

Fonte: Junta Médica do Hospital da Polícia Militar de Santa Catarina - HPM, 2012. 


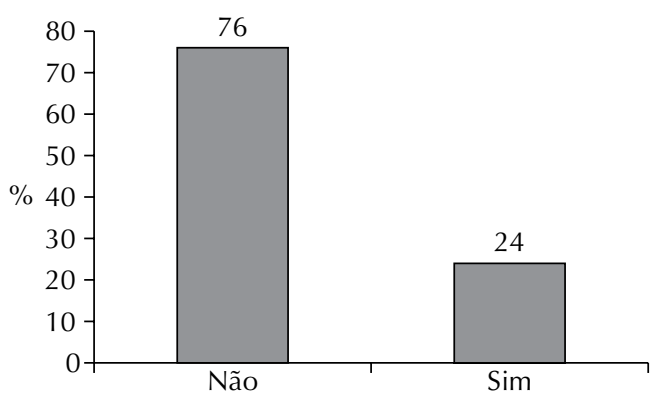

Figura. Prevalência de Transtorno Mental e Comportamental em Policiais Militares da região Metropolitana de Florianópolis/SC, em licença para tratamento de saúde no ano de 2012. Casos notificados.

O conjunto de dados para idade, tempo de serviço e local de trabalho foi agrupado com o objetivo de tornar a distribuição mais homogênea. As variáveis sexo e hierarquia entraram na modelagem. No modelo, hierarquia apresentou significância, ou seja, é fator de associação positiva com TMC e a variável sexo não apresentou significância (Tabela 2 e Tabela 3).

\section{Discussão}

A predominância do sexo masculino está presente em todos os estudos que verificaram esta variável em policiais militares. Este resultado está diretamente relacionado ao processo de ingresso na Polícia Militar. Os próprios editais de concurso para a carreira deixam claro que o número de vagas para mulheres é consideravelmente inferior ao das vagas destinadas aos homens.

No estudo realizado com policiais militares da cidade do Rio de Janeiro, a porcentagem para homens é de $96,3 \%$ e a faixa etária, compreendida entre 31 e 40 anos, com 43,1\% (Souza, Minayo, Silva \& Pires, 2012). No estudo realizado no Recife/PE, a média de idade foi de 38 anos. A faixa etária de policiais militares da região metropolitana de Florianópolis com maior frequência está entre 40 e 49 anos (46\%), deferindo de outros estudos. Esta diferença pode estar relacionada à abrangência da pesquisa, ou seja, diferença entre policiais exercendo a atividade de

Tabela 2. Variáveis associadas com Transtorno Mental e Comportamental, obtidas por meio do Teste do Qui-Quadrado de Pearson $\left(\chi^{2}\right)$. Policiais Militares da região metropolitana de Florianópolis/SC, em licença para tratamento de saúde. Casos Notificados no ano de 2012, $(n=48)$.

\begin{tabular}{|c|c|c|c|c|}
\hline Variável & $\mathbf{n}$ & $\%$ & $\mathbf{p}\left(\chi^{2}\right)$ & OR bruto (IC95\%) \\
\hline \multicolumn{5}{|l|}{ Sexo } \\
\hline Masculino & 41 & 22,8 & 0,20 & $1,82(0,68 ; 4,87)$ \\
\hline Feminino & 7 & 35,0 & & \\
\hline \multicolumn{5}{|l|}{ Idade } \\
\hline De 18 a 39 anos & 24 & 25,0 & 0,75 & $1,11(0,58 ; 2,12)$ \\
\hline De 40 a 59 anos & 24 & 23,1 & & \\
\hline \multicolumn{5}{|l|}{ Tempo de serviço } \\
\hline De 6 meses a 10 anos & 14 & 24,1 & 0,95 & 1 \\
\hline De 11 a 20 anos & 15 & 22,7 & 0,90 & $1,04(0,47 ; 2,31)$ \\
\hline 21 anos ou mais & 19 & 25,0 & 0,75 & $1,13(0,52 ; 2,46)$ \\
\hline \multicolumn{5}{|l|}{ Batalhões } \\
\hline Batalhões comuns & 32 & 26,7 & 0,19 & 1 \\
\hline Batalhões especializados & 1 & 5,3 & 0,76 & $0,89(0,44 ; 1,82)$ \\
\hline Outros & 15 & 24,6 & 0,09 & $5,87(0,72 ; 47,75)$ \\
\hline \multicolumn{5}{|l|}{ Hierarquia } \\
\hline Praça & 43 & 22,2 & 0,01 & $17,55(1,99 ; 154,33)$ \\
\hline Oficial & 5 & 83,3 & & \\
\hline
\end{tabular}


Tabela 3. Regressão logística multivariada para as variáveis associadas ao TMC. Policiais Militares da região metropolitana de Florianópolis/SC, em licença para tratamento de saúde. Casos Notificados no ano de 2012, $(\mathrm{n}=48)$.

\begin{tabular}{lcccccc}
\hline Variável & $\mathbf{n}$ & $\mathbf{\%}$ & OR bruto (IC95\%) & $\mathbf{p}$ & OR ajust. (IC95\%) & $\mathbf{p}$ \\
\hline Hierarquia & & & & & & \\
$\quad$ Praça & 43 & 22,2 & 1 & 1 & \\
$\quad$ Oficial & 5 & 83,3 & $17,55(1,99 ; 154,33)$ & 0,01 & $20,58(2,19 ; 192,80)$ & 0,008 \\
Batalhões & & & & & 1 & \\
$\quad$ Batl. comuns & 32 & 26,7 & 1 & & $1,65(0,73 ; 3,69)$ & 0,22 \\
$\quad$ Outros & 15 & 24,6 & $5,87(0,72 ; 47,75)$ & 0,09 & 1 & \\
Sexo & & & & & & \\
$\quad$ Masculino & 41 & 22,8 & 1 & & $0,52(0,18 ; 1,53)$ & 0,23 \\
$\quad$ Feminino & 7 & 35,0 & $1,82(0,68 ; 4,87)$ & 0,20 & 0
\end{tabular}

$p$ do modelo $(p=0,0001)$. Significância do Teste de Hosmer-Lemeshow $(p=0,976)$.

Ajustado por batalhão e sexo.

trabalho, e este estudo que incluiu apenas policiais militares em LTS.

Pesquisa realizada para verificar a prevalência de TMC em policiais civis de Santa Catarina em LTS nos anos de 2009 e 2010, observou que a média de idade foi de 45,5 anos, corroborando os dados encontrados neste estudo com Policiais Militares. No entanto, os resultados diferem dos encontrados nesta pesquisa em relação à frequência de afastamentos entre os sexos, uma vez que na Polícia Civil os resultados foram equilibrados: sexo feminino, com $51,3 \%$, e sexo masculino, com 48,6\% (Castro, 2012).

Sobre a prevalência de TMC notificado, o maior índice foi relacionado ao bloco F40-48, Transtornos neuróticos, relacionados ao estresse e somatoformes, especificamente, o código F-43 (reação à estresse grave e transtornos de ajustamento) foi o que apresentou maior prevalência. Segundo a Classificação deTranstornos Mentais e de Comportamento da CID-10 (Organização Mundial da Saúde, 1993), "os transtornos agrupados nessa categoria são supostos como surgindo sempre como uma consequência direta de grave estresse agudo ou de trauma continuado" (p.143). Em segundo lugar, sobressai-se o bloco F30-39 Transtornos do humor (afetivos) e, especificamente, o código F-32 Episódio depressivo, "o indivíduo usualmente sofre de humor deprimido, perda de interesse e prazer e energia reduzida levando a uma fatigabilidade aumentada e atividade diminuída" (p. 117).

Estudo realizado por Castro (2012) foi a única pesquisa brasileira com o objetivo de verificar a prevalência de TMC em policiais em LTS. Estudos sobre afastamento do trabalho por transtornos mentais em outras categorias profissionais são mais frequentes (Braga, Carvalho, \& Binder, 2010; Carlotto, Amazarray, Chinazzo, \& Taborda, 2011; De Marco, Citero, Moraes, \& Nogueira-Martins, 2008; Kirchhof et al., 2009; Souza, Carvalho, Araújo, \& Porto, 2010).

Dados brutos da Polícia Militar da Paraíba, 2003 a 2005, demonstram afastamentos análogo nos três anos. No ano de 2003, houve 494 LTS, em 2004, 482 LTS e, em 2005, um número de 491 LTS. São dados brutos, que não falam de prevalência, mas indicam que não houve uma redução significativa, e sim uma estagnação no número de afastamentos. Este quadro é preocupante, pois pode significar que nenhuma medida está sendo tomada para a minimização desses dados e para a melhoria da saúde mental destes profissionais. Outra informação desse estudo, refere-se à aposentadoria por invalidez, constatando que oTMC apresentou maior frequência nos três anos, representando $42 \%$, $27,5 \%$ e $27 \%$, respectivamente, das aposentadorias (Silva, 2007). 
O sofrimento psíquico está diretamente relacionado à saúde mental destes profissionais, às condições de trabalho relacionadas ao constante estado de alerta e a disponibilidade as situações de riscos que a profissão exige, alicerçam a relevância para o fortalecimento da produção científica sobre a temática, no entanto, não é observada uma constância de estudos (Silva, 2009). A prevalência de sofrimento psíquico em policiais militares da cidade do Rio de Janeiro apresentou um índice de em $35,7 \%$. O sofrimento psíquico pode ser considerado indício de algum transtorno mental e comportamental (Souza et al., 2012).

$\mathrm{Na}$ literatura pesquisada, observa-se que a principal temática estudada com policiais militares é a relação destes profissionais com o estresse (Oliveira, \& Bardagi, 2010; Costa et al., 2007). Oliveira e Bardagi (2010) encontraram prevalência de $57,3 \%$ dos participantes com sintomas de estresse em policiais militares da cidade de Santa Maria/RS. Os policiais militares estão propensos ao estresse, devido a contínua tensão e ao risco inerente a profissão (Costa, Accioly Júnior, \& Maia, 2007).

$\mathrm{Na}$ CID-F existem os transtornos relacionados ao estresse, nesse sentindo, o elevado número de policiais com sintomatologia de estresse, especificamente os relacionados ao policiamento ostensivo, é esperado, pois é uma atividade de constante relação com a criminalidade e exposição a riscos. É importante estar atento aos sintomas de estresse, uma vez que esteTMC influencia a condição de trabalho do policial militar e pode causar possíveis prejuízos à sociedade. Administrar esta demanda se torna fundamental para estes profissionais (Oliveira, \& Bardagi, 2010).

No entanto, deve-se ressaltar que número corresponde à frequência de TMC notificado, ou seja, deve haver profissionais atuando mesmo com o transtorno instalado, mas que optam em não se afastarem do trabalho por diversas motivações, sejam elas financeiras ou pessoais. Baierle e Merlo (2008) reforçam esta ideia, afirmando que o desgaste da profissão pode ser negado e, assim, trabalhar doente devido a perdas e possíveis discriminações que podem ocorrer com seu afastamento do trabalho.
No mapeamento das fontes de estresse em Profissionais da Segurança Púbica de Santa Catarina (2010), apresentou-se como conclusão que as principais fontes de estresse ocupacional estão relacionados as condições e organização de trabalho. $\mathrm{O}$ fato de realizarem atividade extra para complemento da renda, carga horária de trabalho, como plantões, "percepção de que a eficácia do trabalho é comprometida nas relações com a Justiça" (p. 50), são entendidos como fatores estressores.

Silva (2009) acrescenta a ideia de que o sofrimento psíquico pode surgir através da somatização, e essa relação nem sempre é percebida pelo indivíduo. Ou seja, LTS com outros diagnósticos podem conter causa principal a doença mental e, dessa forma, não entrar nos índices de prevalência de TMC.

Na comparação sobre a associação entre as variáveis demográficas e de vínculo com a corporação com o Transtorno Mental e Comportamental em Policiais Militares, observa-se o estudo realizado por Souza et al. (2012) que descrevem que o maior índice de sofrimento psíquico, $24 \%$ dos policiais militares do Rio de Janeiro, estão entre 11 e 20 anos de trabalho, seguido por $16,2 \%$ dos policiais com mais de 20 anos de serviço. No referido estudo, Souza et al. (2012) evidenciaram que as variáveis socioeconômicas e demográficas não apresentam relação com o sofrimento psíquico, e que a insatisfação com inabilidade frente a situações difíceis e a outras questões relacionadas a sua vida pessoal apresentam maior evidência para o sofrimento psíquico.

Ainda sobre a relação entre tempo de serviço e frequência de afastamento por TMC, estudo com Policiais Civis de SC, nos anos de 2009 e 2010, realizado por Castro (2012), constata uma oscilação significativa aos 5 , 15 e 25-26 anos. Esses dados reforçam os resultados encontrados neste estudo, em que a frequência de LTS com diagnósticos de TMC foi maior em indivíduos com tempo de serviço entre 5 a 10 anos (34,1\%), seguido pelo tempo de serviço de 11 a 15 anos (30\%). 
Segundo Ferreira, Bonfim, \& Augusto (2012), foi alto o índice de morbidade autoreferido por Policiais Militares da cidade do Recife/PE, especialmente os relacionados ao nível de estresse. Outros autores apresentam resultados semelhantes relacionados aos sintomas de estresse (Costa et al., 2007; Dantas et al., 2010; Minayo et al., 2008). Minayo et al. (2011) observaram elevado índice de sofrimento psíquico entre Policiais Militares $(33,6 \%)$, em um estudo no Rio de Janeiro/RJ, quando comparados a Policiais Civis (20,3\%). Os referidos autores relacionam o sofrimento psíquico com sintomas psicossomáticos, depressivos e de ansiedade e referem sobre o alto número de licenças por questões psiquiátricas, mesmo havendo resistência das chefias em liberar para o tratamento para estes diagnósticos.

Quanto à associação encontrada neste estudo, a categoria definida como Oficial, apresentou mais chance de ter Transtorno Mental e Comportamental do que a categoria definida como Praças. Os oficiais, como definem Minayo et al. (2008), em sua maioria são responsáveis por organizar e planejar as estratégias de ação para as diversas situações, sejam elas operações de baixo ou alto nível de risco.

Costa et al. (2007) estudaram o estresse em policiais militares da cidade de Natal/RN, em uma amostra estratificada por grupos hierárquicos. A prevalência de estresse foi de $47,4 \%$, número que os autores consideraram preocupante. No entanto, diferentemente do encontrado neste estudo com policiais militares em LTS, o percentual de indivíduos com estresse entre Oficiais $(55,6)$ e Praças $(49,5)$ foram semelhantes. Oliveira e Bardagi (2010), em estudo com Polícia Militar de Santa Maria/RS, descreveram a frequência de estresse em três áreas desta corporação, $65,4 \%$ pertenciam ao grupo de atendimento de emergência (CRE/Copom), 57,1 no grupo de policiamento ostensivo e apenas $28,6 \%$ no grupo do administrativo.

No estudo já citado anteriormente, Minayo et al. (2008) encontraram uma frequência de lesões físicas permanentes em Oficiais $(21,3 \%)$, quando comparados aos Praças
$(12,7 \%)$ e quando comparados especificamente as lesões com o trabalho, há uma associação para $17,7 \%$ dos oficiais, suboficiais e sargentos e de 9,7\% para os soldados e cabos. Ainda neste estudo, os autores relatam que o estresse ocupacional tem relação com o poder de decisão que as chefias possuem. Os Oficiais, responsáveis pelas atividades de comando, descrevem um estresse frequente e constante devido à pressão dos altos escalões responsáveis pela Segurança Pública, pelos excessos que a mídia comete e, em consequência, o reconhecimento da sociedade e a responsabilidade de planejamento das ações e dos subordinados envolvidos nas operações. Os resultados da regressão logística parecem suportar esta hipótese, ou seja, esta alta demanda de risco que o Oficial tem que dar conta pode estar relacionado ao afastamento do trabalho com diagnósticos relacionados à saúde mental deste trabalhador. A própria legislação militar é mais severa com os oficiais, pois considera essa condição como um agravante para a ocorrência vinculada a essa legislação.

Profissionais de outras áreas que exercem atividades de gestão que exigem decisões e ações rápidas também trabalham sobre pressão, e suas ações também tem efeito sobre a vida de outras pessoas. Estudo realizado sobre o estresse em executivos de grandes empresas do Brasil (Mota, Tanure, \& Carvalho Neto, 2008) aponta que há uma dificuldade para esses profissionais se desligarem do trabalho, devido à carga de responsabilidade diária, metas e a serem atingidas, competitividade e, assim, tornando-se mais suscetível ao estresse.

O estresse é um tema bastante discutido quando relacionado aos cargos gerenciais e saúde mental. Pressões inerentes ao cargo influenciam diretamente a saúde mental destes profissionais. Estudo sobre os envolvidos no corpo gerencial de uma empresa pública de Minas Gerais descreve desgastes físicos quanto mentais devido a processo de reestruturação que a Empresa estava passando. Adaptar as novas necessidades ocasiona um nível elevado de estresse sobre os gestores, pois eles executam uma importante ligação entre a alta direção e o nível operacional, 
organizando e alinhando todos os processos para a nova realidade. No referido estudo, observa-se que $56 \%$ descrevem como elevado o tipo de pressão que sofrem devido ao papel gerencial que exercem, ou seja, apenas o fato de ter este papel de gerente já representa alta fonte de pressão, pois são cobrados para a absoluta eficiência (Paiva, \& Couto, 2008). Em estudo com pessoas em posições de liderança feito em uma empresa de energia elétrica, os estressores foram categorizados em três: relativos à organização, às tarefas ou às pessoas. Os autores descrevem que lidar com as tarefas e as pessoas aapresenta frequência elevada para os as aspectos estressores. Verifica-se também que, quanto mais experiente e maior o tempo de trabalho no papel de liderança, melhor a maneira de enfrentar os aspectos estressores (Fonte, Neri, \& Yassuda, 2010).

No entanto, por se tratar de empresa privada, estratégias de valorização da mão de obra madura pode ser uma prioridade. Aspecto diferente da Segurança Pública, pois são profissionais de carreira que vão galgando postos elevados hierarquicamente conforme o tempo de serviço e, nesse sentido, as estratégias de enfrentamento dos estressores deve levar isso em consideração.

Lipp (2001) apresenta um levantamento sobre estresse realizado na cidade de Campinas (SP) em que $70 \%$ das pessoas que procuraram atendimento no Centro Psicológico de Controle do Stress estavam na categoria profissional relacionada a classe gerencial. Bernik (apud Lipp, 2001) ressalta que os executivos pertencem a uma das categorias mais relacionadas ao estresse e fatores como instabilidade econômica e política.

Estudos comparando os cargos exercidos com o estresse ou outroTMC são escassos, conforme relatam Sadir e Lipp (2009). Em função disso, sugere-se que novos estudos sejam realizados para verificar oTMC numa instância ampliada para aprofundar a questão de diferentes cargos nas mais diversas profissões.

Estudo sobre a saúde mental de policiais na Paraíba demonstra um caminho diferente do encontrado neste estudo, como relata Silva e Vieira (2008) que descrevem $73,2 \%$ dos aposentados por invalidez por transtornos psiquiátricos sendo os soldados e cabos. Os autores relatam ainda que "sejam eles civis ou militares, os policiais em atividade-fim caminham, na maioria das vezes, no limiar entre a vida e a morte" (p. 166). Na Polícia Militar da Paraíba, há preocupação das lideranças sobre o elevado número de policiais envolvidos com a dependência química, especificamente o álcool, frequente estresse, casos de depressão e até mesmo suicídio entre os policiais militares. As hipóteses para o aumento da demanda negativa na saúde mental dos policiais militares são a elevada pressão da organização de trabalho e a pressão da sociedade com estes profissionais (Silva, \& Vieira, 2008).

Silva (2007) reforça a ideia de um diálogo entre a academia e a Polícia Militar, fundamentando parcerias na construção de metodologias e procedimentos pensando na qualidade de vida destes profissionais.

\section{Conclusão}

Este estudo buscou verificar a prevalência de Transtorno Mental e Comportamental em Policiais Militares da região metropolitana de Florianópolis/SC, em Licença para Tratamento de Saúde, no ano de 2012.

Observou-se neste estudo associação de transtorno mental e comportamental em policiais militares na categoria de oficias, podendo ser devido aos cargos de chefias e da responsabilidade desta categoria hierárquica, ou seja, o alto grau de responsabilidade que suas decisões demandam, a preocupação em liderar um contingente grande de pessoas. $\mathrm{E}$ isso envolve o impacto direto na integridade física dos envolvidos, na segurança e na própria vida de seus subordinados.

Os resultados encontrados evidenciam a necessidade de atenção específica para a demanda psicológica de Policiais Militares. Podemos considerar a falta de pesquisas voltadas para o estudo de transtorno mental e 
comportamental em policiais militares de Santa Catarina como uma limitação que impossibilita a comparação com outros resultados. A maioria dos estudos é voltada especificamente às questões relacionadas ao estresse, em outros estados, e com policiais exercendo a atividade de trabalho normalmente. Outra limitação encontrada foi a falta de um banco de dados informatizado e padronizado para a coleta de dados.

Desta forma, compreender e analisar estes dados contribuirá para o desenho de estratégias de prevenção e de enfrentamento ao TMC, que poderão melhorar a qualidade de vida de Policiais Militares de Santa Catarina. Ao se estimar a prevalência de TMC e verificar possíveis associações, possibilitar-se-á a adoção de medidas que visem à diminuição de índices de afastamento do trabalho e de absenteísmo e fornecer elementos para orientação dos investimentos de recursos em programas que preservem a saúde psicológica desta categoria. E, numa instância ampliada, favorecer a população que depende do fazer destes profissionais.

Ressalta-se que essa pesquisa busca ampliar o conhecimento já produzido, considerando-se as especificidades profissionais dos participantes do estudo. 


\section{Fabíola Polo de Lima}

Mestre em Saúde Coletiva pela Universidade Federal de Santa Catarina - SC. Brasil. E-mail: fabiolapolo@gmail.com

\section{Vera Lúcia Guimarães Blank}

Doutora em Medicina Social pelo Instituto Karolinska. Docente Colaboradora do Departamento de Saúde Pública pela Universidade Federal de Santa Catarina - SC. Brasil. E-mail: vblank@ccs.ufsc.br

\section{Fabricio Augusto Menegon}

Doutor em Saúde Pública pela Universidade de São Paulo - SP. Professor adjunto do Departamento de Saúde Pública da Universidade Federal de Santa Catarina - SC. Brasil. E-mail: f.menegon@ufsc.br

\section{Endereço para envio de correspondência:}

Rua Acelon Pacheco da Costa, 231 - apto 203A. CEP: 88034-040 Florianópolis - SC. Brasil.

Recebido: 26/09/2013, Aprovado: 03/07/2015. 
Andrade, E. R., Souza, E. R., \& Minayo, M. C. S. (2009). Intervenção visando a auto-estima e qualidade de vida dos policiais civis do Rio de Janeiro. Ciência \& Saúde Coletiva, 14(1), 275-285.

Asmus, C.I. R. F., Meyer, A., \& Castro, H.A. (2009). Epidemiologia e saúde do trabalhador. In Medronho, R. A. Epidemiologia. São Paulo, SP: Atheneu.

Baierle, T., \& Merlo, A. (2008). Saúde mental e subjetividade no trabalho de uma guarda municipal: estudo em psicodinâmica do trabalho. Cadernos de Psicologia Social do Trabalho, 11(1), 69-81. doi:10.11606/issn. 1981-0490.v11i1p69-81

Bezerra, C. M., Minayo, M. C. S. \& Constantino, P. (2013). Estresse ocupacional em mulheres policiais. Ciência \& Saúde Coletiva, 18(3), 657-666. doi:10.1590/S1413-81232013000300011

Braga, L. C., Carvalho, L. R., \& Binder, M. C. P. (2010). Condições de trabalho e transtornos mentais comuns em trabalhadores da rede básica de saúde de Botucatu (SP). Ciência \& Saúde Coletiva, 15(supl 1), 1585-1596. doi:10.1590/S1413-81232010000700070

Brasil. (2014a). Ministério da Previdência Social. Principais causas de afastamento do trabalho entre homens e mulheres empregados da iniciativa privada. Brasília, DF: o autor. (2 Boletim Quadrimestral sobre benefícios por Incapacidade). Recuperadoem10dedezembro de 2014, de http://www.previdencia.gov.br/ wp-content/uploads/2015/01/2\%C2\%BAboletim-quadrimestral1.pdf

Brasil. (2014b). Ministério da Previdência Social. Acompanhamento mensal dos benefícios auxílios-doença previdenciários concedidos segundo os códigos da CID-10: janeiro a abril de 2014. Brasília, DF: o autor. Recuperado em 10 de dezembro de 2014, de http://www.previdencia.gov.br/wp-content/ uploads/2014/06/Aux-Doenca-Conc-Preve-Acid-X-CID-e-Clientela_2014_separadoate-ABR-previdenciarios.pdf

Browers, E. P. M., Terluin, B., Tiemens, B. G., \& Verhaak, P. F.M. (2009). Predicting return to work in employees sick-listed due to minor mental disorders. Journal of Occupational Rehabilitation, 19, 323-332. doi:10.1007/s10926-009-9198-8
Campos, I.C.M. (2006). Diagnósticos de transtornos mentais e comportamentais e relação com o trabalho de servidores públicos estaduais. Dissertação de Mestrado, Universidade Federal de Santa Catarina, Florianópolis, SC.

Carlotto, M.S., Amazarray, M.R., Chinazzo, I., \& Taborda, L. (2011). Transtornos mentais comuns e fatores associados em trabalhadores: uma análise na perspectiva de gênero. Cadernos de Saúde Coletiva, 19(2), 172-178.

Castro, M.C.A. (2012). Prevalência de transtornos mentais e comportamentais e percepção de suporte familiar em policiais civis. Dissertação de Mestrado, Universidade Federal de Santa Catarina, Florianópolis, SC.

Costa, M., Accioly Júnior, O., \& Maia, E. (2007). Estresse: diagnóstico dos policiais militar em uma cidade Brasiléia. Revista Panamericana de Salud Publica, 21(4): 217-22. doi:10.1590/S1020-49892007000300004

Costa, S. H. N., Cunha, L. C., Yonamine, M., Pucci, L. L., Oliveira, F. G. F., Souza, C. G., et al. (2010). Survey on the use of psychotropic drugs by twelve military Police units in the municipalities of Goiânia and Aparecida de Goiânia, state of Goiás, Brazil. Revista Brasileira de Psiquiatria, 32(4), 389-395. doi:10.1590/S1516-44462010005000023

Cruz, R. M. (2010). Nexo técnico e vigilância à saúde do trabalhador: uma agenda científica para o NTEP. In: J Machado, L. Soratto, \& W. Codo (Org.), Saúde e trabalho no Brasil: uma revolução silenciosa. Petrópolis, RJ: Vozes.

Dantas, M. A., Brito, D. V. C., Rodrigues, P. B., \& Maciente, T. S. (2010). Avaliação de estresse em policiais militares. Psicologia: Teoria e Prática, 12(3): 66-77.

De Marco, P. F., Citero, V. A., Moraes, E., \& Nogueira-Martins, L. A. (2008). O impacto do trabalho em saúde mental: transtornos psiquiátricos menores, qualidade de vida e satisfação profissional. Jornal Brasileiro de Psiquiatria, 57(3): 178-183. doi:10.1590/S0047-20852008000300004

Ferreira, D. K., Augusto, L. G. S., \& Silva, J. (2008). Condições de trabalho e percepção da saúde de policiais militares. Cadernos de Saúde Coletiva, 16(3), 403-420. 
Ferreira, D. K., Bonfim, C., \& Augusto, L. G. S. (2012). Condições de trabalho e morbidade referida de policiais militares, Refice/PE, Brasil. Saúde e Sociedade, 21(4), 939-1000. doi:10.1590/S0104-12902012000400016

Fonte, A. P., Neri, A. L., \& Yassuda, M. S. (2010) Enfrentamento de estresse no trabalho: relações entre idade, experiência, autoeficácia e agência. Psicologia, Ciência e Profissão, 30(3), 620-633. doi:10.1590/S1414-98932010000300013

Kirchhof, A. L. C., Magnano, T. S. B. S., Camponogora, S., Griep, R. H., Tavares, J. P., Prestes, F. C. et al. (2009). Condições de trabalho e características sócio-demográficas relacionadas à presença de distúrbios psíquicos menores em trabalhadores de enfermagem. Texto e Contexto - Enfermagem, 18(2): 215-223. doi:10.1590/S0104-07072009000200003

Lipp, M. E. N. (2001). Stress emocional: a contribuição de estressores internos e externos. Psiquiatria Clínica, 28(6): 347-349.

Mascarenhas, R. B. (2009). As condições e organização das atividades profissionais do policial militar de Goiás e as repercussões em sua saúde mental: 2005 a 2007. Dissertação de Mestrado, Universidade Católica de Goiás, Goiania, GO.

Mendes, A. M., Ghizoni, L. D., \& Araújo, L. K. R. (2011). Diagnósticos dos riscos no trabalho para os transtornos psicossociais nos diferentes setores da indústria. Brasília, DF: SESI.

Minayo, M. C. S., Souza, E. R., Constantino, P. (Coord). (2008). Missão prevenir e proteger: condições de vida, trabalho e saúde dos policiais militares do Rio de Janeiro. Rio de Janeiro, RJ: Fiocruz.

Minayo, M. C. S., Souza, E. R., \& Constantino, P. (2007). Riscos percebidos e vitimização de policiais civis e militares na (in) segurança pública. Cadernos de Saúde Pública, 23(11), 2767-2779. doi:10.1590/S0102-311X2007001100024

Minayo, M.C.S, Assis, S.G. \& Oliveira, R.V.C. (2011). Impacto das atividades profissionais na saúde física e mental dos policiais civis e militares do Rio de Janeiro (RJ, Brasil). Ciência \& Saúde Coletiva, 16(4): 2199-2209 doi:10.1590/S1413-81232011000400019
Mota, C. M., Tanure, B., \& Carvalho Neto, A. (2008). Estresse e sofrimento no trabalho dos executivos. Psicologia em Revista, 14(1), 107-130.

Oliveira, P. L. M \& Bardagi, M. P. (2010). Estresse e comprometimento com a carreira em policiais militares. Boletim de Psicologia, 59(131), 153-166.

Organização Mundial de Saúde. (2000). CID-10 classificação estatística internacional de doenças e problemas relacionados à saúde (10a ed. rev.). São Paulo, SP: Universidade de São Paulo.

Organização Mundial de Saúde. (1993). CID-10 Classificação de transtornos mentais e de comportamento da CID-10: descrições clínica e diretrizes diagnósticas. Porto Alegre, RS: Artmed.

Paiva, K. C. M., \& Couto, J. H. (2008). Qualidade de vida e estresse gerencial "pós-choque de gestão": o caso da Copasa-MG. Revista de Administração Pública, 42(6), 1189-1211.

Polícia Militar de Santa Catarina. Portal da Polícia Militar de Santa Catarina. Institucional. História da Polícia Militar de Santa Catarina. Recuperado em 13 de junho de 2011, de http://www.pm.sc.gov.br/website/redir. php? site $=40 \&$ act $=1 \& \mathrm{id}=4 \&$ url $=4$

Roelen, C. A. M., Koopmans, P. C., Anema, J. R., \& Beek, A. J. V. (2010). Recurrence of medically certified sickness according to diagnosis: a sickness absence register study. Journal of Occupational Rehabilitation, 20(1), 113-121. doi:10.1007/s10926-009-9226-8

Sadir, M. A., \& Lipp, M. E. N. (2009). As fontes de stress no trabalho. Revista de Psicologia da IMED, 1(1), 114-126.

Santa Catarina, (2010), Governo do Estado. Lei Complementar Estadual $\mathrm{n}^{\circ} 495$, de 26 de janeiro de 2010. Regiões Metropolitanas de Santa Catarina.

Santana, V. S. (2006). Saúde do trabalhador no Brasil: pesquisa na pós-graduação. Saúde Pública, 40(N Esp):101-11.

Silva, J. H. R. (2009). Estudo sobre o trabalho do policial e suas implicações na saúde mental. Dissertação de Mestrado. Universidade de São Paulo, São Paulo, SP. 
Silva, M. B. (2007). Trabalho de polícia militar e saúde mental: um estudo de caso sobre sofrimento psíquico e prazer na atividade de radiopatrulha. Dissertação de Mestrado. Universidade Federal da Paraíba, João Pessoal, PB.

Silva, M., \& Vieira, S. (2008). O processo de trabalho do militar estadual e a saúde mental. Saúde e Sociedade, 17(4), 161-170. doi:10.1590/S0104-12902008000400016

Souza, E. R., \& Minayo, M. C. S. (2005). Policial, risco como profissão: morbimortalidade vinculada ao trabalho. Ciência \& Saúde Coletiva, 10(4), 917-928.

Souza, E. R., Minayo, M. C. S., Silva, J. G., \& Pires, T. O. (2012). Fatores associados ao sofrimento psíquico de policiais militares da cidade do Rio de Janeiro, Brasil. Cadernos de Saúde Pública, 28(7), 1297-1311. doi:10.1590/S0102-311X2012000700008
Souza, E. R., Schenker, M., Constantino, P. \& Correia, B. S. C. (2013) Consumo de substâncias lícitas e ilícitas por policiais da cidade do Rio de Janeiro. Ciência \& Saúde Coletiva, 18(3), 667-676. doi:10.1590/S1413-81232013000300012

Souza, S. F., Carvalho, F. M., Araújo, T. M., \&, Porto, L. A. (2010) Fatores psicossociais do trabalho e transtornos mentais comuns em eletricitários. Revista de Saúde Pública, 44(4), 770-117. doi:10.1590/S0034-89102010000400015

VandenBos, G. R. (2010). Dicionário de Psicologia da APA. Porto Alegre, RS: Artmed.

World Health Organization. (2001). The World health report, 2001: mental health, new understanding, new hope. Geneva: the author.

World Health Organization. (2011). World Health Statistics 2011. Geneva: the author. 